УДК 621.397, 004.421

\title{
ХАРАКТЕРИСТИКИ ДВУМЕРНОЙ ОЦЕНКИ ПОЛОЖЕНИЯ ФРАГМЕНТА ИЗОБРАЖЕНИЯ НА ОСНОВЕ АЛГОРИТМА НЬЮТОНА - РАФСОНА
}

\author{
Ю. С. Радченко, О. А. Машарова \\ Воронежский государственный университет
}

Поступила в редакцию 16.10.2018 г.

\begin{abstract}
Аннотация. В работе рассмотрена двумерная оценка положения фрагмента изображения в кадре с помощью алгоритмов типа Ньютона - Рафсона. Данные алгоритмы реализованы в виде некоторых типов дискриминаторов. Рассмотрена двумерная дискретная автокорреляционная функция тестового объекта и определены условия ее факторизации на одномерные составляющие. Для некоторых типов дискриминаторов исследовано поведение дискриминационной характеристики. Показано, что плотность вероятности процесса на выходе дискриминатора существенно негауссовская с «тяжелыми хвостами». Методами статистического моделирования подтверждены теоретические расчеты.

Ключевые слова: оценка положения фрагмента изображения, дискриминаторы, негауссовское распределение статистики, «тяжелые хвосты распределения», статистическое моделирование.

Annotation. This paper considers a two-dimensional estimation of fragment position of image in a frame by using Newton - Rafson type algorithms. These algorithms are implemented as some types of discriminators. Considered a two-dimensional discrete autocorrelation function of test object and the conditions of its factorization to one-dimensional components are defied. The behavior of discriminator characteristics were analyzed for some types of discriminators. It is shown that probability density of process on discriminator output is substantially non-Gaussian with «heavy tails». By using statistical modeling the theoretical calculations are confirmed.

Keywords: estimation of fragment position of image, discriminators, non-Gaussian distribution of statistics, «heavy tails of distribution», statistical modeling.
\end{abstract}

\section{ВВЕДЕНИЕ}

При видеокодировании, интеллектуальном видеонаблюдении, и в других приложениях возникает задача оценки местоположения фрагмента или блока изображения в кадре [1, $2,3,4,5]$. В современных системах точность, с которой необходимо оценить местоположение фрагмента, составляет $1 / 4$ пикселя $[1,2]$. В случае, если необходимая точность составляет 1 пиксель, последовательность действий следующая: сначала по некоторому шаблону поиска локализуется область экстремума дискретной целевой функции, затем методом последовательного перебора находится точка экстремума с заданной точностью $[1,4,6]$.

При необходимости оценки с субпиксельной точностью последовательность действий

() Радченко Ю. С., Машарова О. А., 2018 заметно усложняется: после локализации области экстремума необходимо провести передискретизацию дискретной целевой функции в локальной окрестности экстремума, затем провести интерполяцию новых отсчётов. В итоге уточнённое положение экстремума находится методом последовательного перебора $[1,6]$. Очевидно, что реализация такого метода достаточно громоздка. В $[7,8,9,10]$ рассмотрена возможность субпиксельного восстановления изображений (сверхразрешения) на основе анализа и оптимального объединения последовательности кадров. Реализация такого алгоритма также весьма трудоемка. С вычислительной точки зрения, более экономичным является способ оценки положения сигнала с помощью дискриминатора, который реализует вычислительный алгоритм Ньютона - Рафсона $[11,12]$. Такой подход требует небольшого числа отсчетов 
Характеристики двумерной оценки положения фрагмента изображения на основе алгоритма ...

целевой функции и выдаёт вещественные значения, с точностью до долей пикселя. Расчету точности оценки непрерывного параметра одномерного сигнала с помощью дискриминатора посвящена работа [13]. Для ряда типичных дискриминационных алгоритмов было показано, что распределение одномерной оценки имеет «тяжелые хвосты».

Целью данной работы является обобщение полученных результатов на случай двумерной оценки по дискретной целевой функции - логарифму функционала отношения правдоподобия. Проведено исследование дискретного поля логарифма отношения правдоподобия, установлены условия факторизации двумерной целевой функции на одномерные. Найдены вероятностные характеристики оценки фрагмента изображения с помощью раздельных дискриминаторов при различных расстройках по параметрам сигнала и приемника, при конечных отношениях сигнал/шум.

\section{1. МАТЕРИАЛЫ И МЕТОДЫ}

Пусть задано двумерное поле $s(\vec{r}, t)$, $\vec{r}=(x, y)$, представляющее собой фрагмент пространственного сигнала в момент $t$ в области $\Omega_{0}$. В момент $t+\Delta t$ в подобласти $\Omega \in \Omega_{0}$ наблюдается поле (анализируемый кадр) $\xi(\vec{r}, t+\Delta t)=s\left(\vec{r}, \vec{l}_{0}, t\right)+n(\vec{r})$, представляющее собой смесь полезного сигнала $s(\vec{r}, \vec{l})$, смещённого на неизвестный вектор $\vec{l}_{0}=\left(l_{0 x}, l_{0 y}\right)$, и некоррелированной помехи $n(\vec{r})$ со спектральной плотностью мощности $N_{0} / 2$. В дальнейшем дискретная переменная времени $t$ не фигурирует и может быть опущена.

Для решения задачи воспользуемся алгоритмом максимального правдоподобия, согласно которому в качестве целевой функции требуется сформировать логарифм функционала отношения правдоподобия (ЛФОП)

$$
\begin{gathered}
M(\vec{l})=\frac{2}{N_{0}} \int_{\Omega} \xi(\vec{r}) s(\vec{r}, \vec{l}) d \vec{r}- \\
-\frac{1}{N_{0}} \int_{\Omega} s(\vec{r}, \vec{l}) s(\vec{r}, \vec{l}) d \vec{r} .
\end{gathered}
$$

Если пренебречь изменением формы сигнала при сдвиге, то второе слагаемое в (1) можно считать не зависящим от $\vec{l}=\left(l_{x}, l_{y}\right)$ и его не учитывать.

Выделим у ЛФОП $M(\vec{l})$ детерминированную и флуктуационную компоненты:

$$
M(\vec{l})=q^{2} S\left(l_{x}-l_{0 x}, l_{y}-l_{0 y}\right)+q N\left(l_{x}, l_{y}\right) .
$$

Здесь $S\left(l_{x}-l_{0 x}, l_{y}-l_{0 y}\right)$ - нормированная автокорреляционная функция (АКФ), $\left|S\left(\vec{l}-\vec{l}_{0}\right)\right| \leq 1$, $N\left(l_{x}, l_{y}\right)$ - нормированная шумовая функция c нулевым средним значением, единичной дисперсией и корреляционной функцией $<N\left(\vec{l}_{1}\right) N\left(\vec{l}_{2}\right)>=S\left(\vec{l}_{1}-\vec{l}_{2}\right), q^{2}=2 E_{S} / N_{0}$ - энергетическое отношение сигнал/шум. Если обозначить интервал корреляции $M(\vec{l})$ как $\left(\tau_{l x}, \tau_{l y}\right)$, то можно записать:

$$
\begin{gathered}
S\left(l_{x}-l_{0 x}, l_{y}-l_{0 y}\right) \equiv \\
\equiv S\left(\frac{l_{x}-l_{0 x}}{\tau_{l x}}, \frac{l_{y}-l_{0 y}}{\tau_{l y}}\right)=S\left(\theta_{x}, \theta_{y}\right), \\
\left(\frac{l_{x}-l_{0 x}}{\tau_{l x}}, \frac{l_{y}-l_{0 y}}{\tau_{l y}}\right)=\left(\theta_{x}, \theta_{y}\right), \\
\left(\frac{l_{f x}-l_{0 x}}{\tau_{l x}}, \frac{l_{f y}-l_{0 y}}{\tau_{l y}}\right)=\left(\theta_{f x}, \theta_{f y}\right), \\
\left(\frac{\Delta_{x}}{\tau_{l x}}, \frac{\Delta_{y}}{\tau_{l y}}\right)=\left(\delta_{x}, \delta_{y}\right), \\
\left(\lambda_{m_{x}}, \lambda_{m_{y}}\right)=\left(\frac{l_{m_{x}}-l_{f_{x}}}{\tau_{l x}}, \frac{l_{m_{y}}-l_{f_{y}}}{\tau_{l y}}\right) .
\end{gathered}
$$

Здесь $\left(l_{m x}, l_{m y}\right) \rightarrow\left(\lambda_{m x}, \lambda_{m y}\right)$ - оценка максимального правдоподобия (ОМП), $\left(l_{f x}, l_{f y}\right)$ параметр опорного сигнала приёмника, $\left(\theta_{f x}, \theta_{f y}\right)$ - нормированное рассогласование по параметру наблюдаемого сигнала и приемника, $\left(\Delta_{x}, \Delta_{y}\right)$ - расстройка по параметру $\left(l_{x}, l_{y}\right)$ каналов дискриминатора. Переобозначим $M(\vec{l}) / q \rightarrow M(\vec{l})$. Соответственно ЛФОП (2) можно записать в виде: $M(\vec{\theta})=q S(\vec{\theta})+N(\vec{\theta})$.

\section{2. РЕЗУЛЬТАТЫ ИССЛЕДОВАНИЙ И ИХ ОБСУЖДЕНИЯ}

Рассмотрим один из возможных видов изображений. На рис. 1а приведено тестовое изображение - городской пейзаж, на котором надо определить местоположение объекта (автомобиль - рис. 16). 


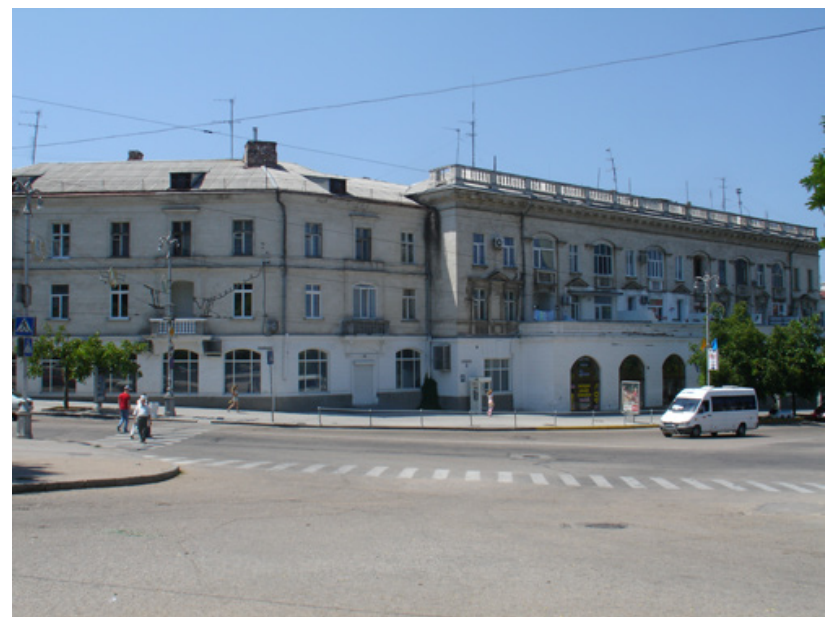

Puc. $1 a$

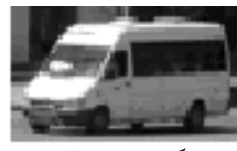

Puc. 16

Анализ АКФ и сравнение ее с модельными объектами показал, что двумерная АКФ в районе максимума может быть факторизована на произведение одномерных АКФ $S\left(\theta_{x}, \theta_{y}\right) \approx S\left(\theta_{x}\right) S\left(\theta_{y}\right)$ по каждой координате $\left(\theta_{x}, \theta_{y}\right)$. Однако, для объектов с произвольным ракурсом такая факторизация напрямую неприменима, поскольку в АКФ присутствуют перекрестные произведения $\left(\theta_{x} \cdot \theta_{y}\right)$. АКФ фрагмента тестового изображения представлена на рис. 2 в двух проекциях.

Для АКФ $S\left(\theta_{x}, \theta_{y}\right)$ имеет место пара преобразований

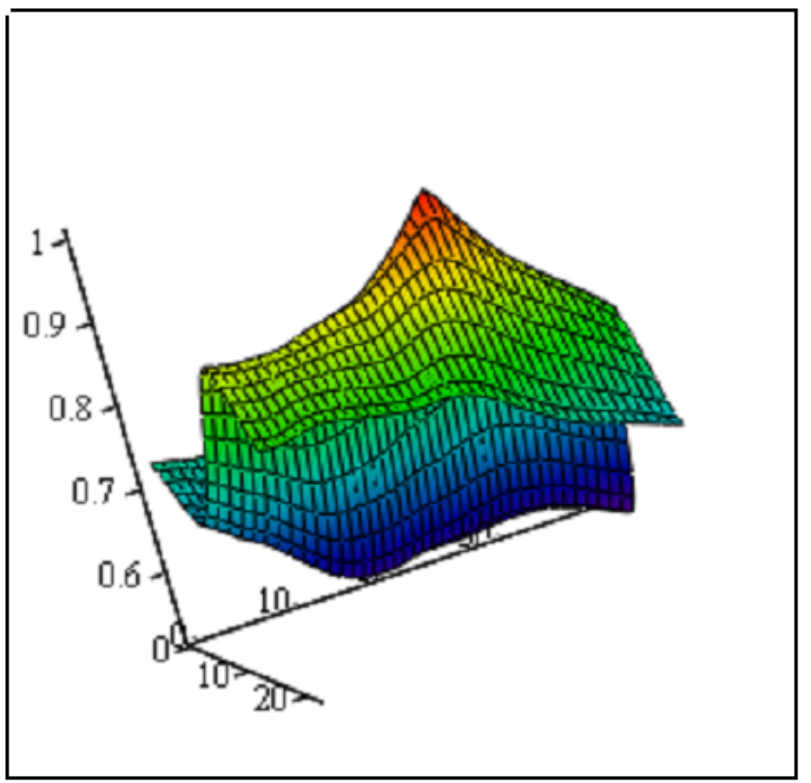

$$
\begin{gathered}
G\left(\omega_{x}, \omega_{y}\right)= \\
=\iint S\left(\theta_{x}, \theta_{y}\right) \exp \left[-j\left(\theta_{x} \omega_{x}+\theta_{y} \omega_{y}\right)\right] d \theta_{x} d \theta_{y}, \\
S\left(\theta_{x}, \theta_{y}\right)=(1 / 2 \pi)^{2} \iint G\left(\omega_{x}, \omega_{y}\right) \times \\
\times \exp \left[j\left(\theta_{x} \omega_{x}+\theta_{y} \omega_{y}\right)\right] d \omega_{x} d \omega_{y},
\end{gathered}
$$

В окрестности положения максимума $\omega_{x}=0$, $\omega_{y}=0$ спектральная плотность $G\left(\omega_{x}, \omega_{y}\right)$, как правило, имеет вид (условие полной линейной регулярности [14])

$$
\begin{gathered}
G\left(\omega_{x}, \omega_{y}\right) \approx \\
\approx G_{00}-0.5\left(G_{11} \omega_{x}^{2}+G_{22} \omega_{y}^{2}+2 G_{12} \omega_{x} \omega_{y}\right),
\end{gathered}
$$

где

$$
\begin{aligned}
& G_{11}=-\partial^{2} G\left(\omega_{x}, \omega_{y}\right) /\left(\partial \omega_{x}\right)^{2}, \\
& G_{22}=-\partial^{2} G\left(\omega_{x}, \omega_{y}\right) /\left(\partial \omega_{y}\right)^{2}, \\
& G_{12}=-\partial^{2} G\left(\omega_{x}, \omega_{y}\right) / \partial \omega_{x} \partial \omega_{y} .
\end{aligned}
$$

Как видно из (4) спектральная плотность в окрестности максимума представляет собой квадратичную форму (в сечении - эллипс). Поворот осей с помощью преобразования

$$
\begin{aligned}
& \theta_{x}=u \cos \varphi-v \sin \varphi \Omega_{u}=\omega_{x} \cos \varphi-\omega_{y} \sin \varphi \\
& \theta_{y}=u \sin \varphi+v \cos \varphi \Omega_{v}=\omega_{x} \sin \varphi+\omega_{y} \cos \varphi
\end{aligned}
$$

приводит квадратичную форму (4) к каноническому виду $[15,16]$

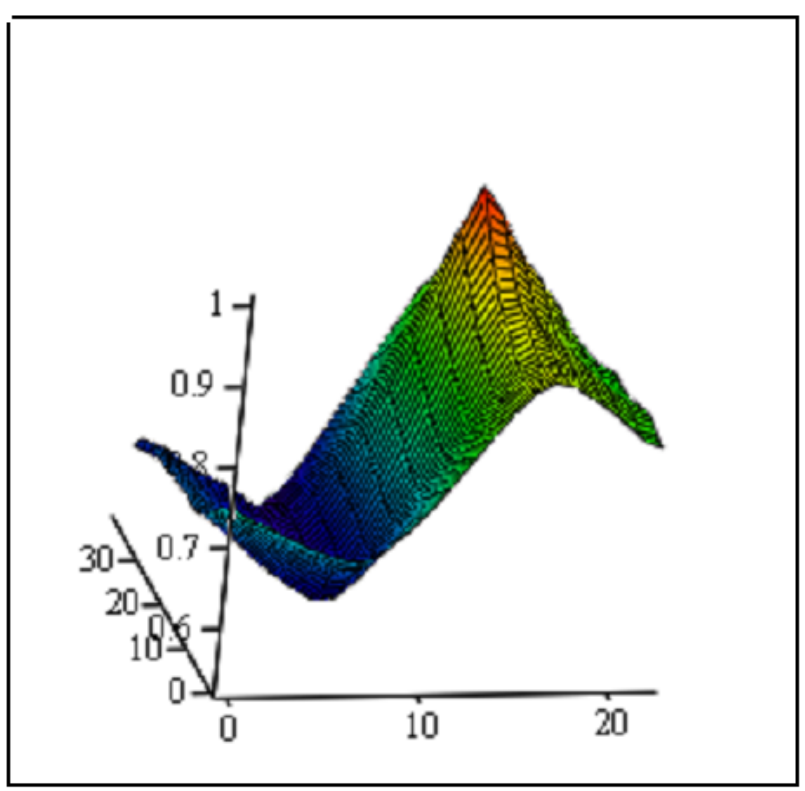

Puc. 2 
Характеристики двумерной оценки положения фрагмента изображения на основе алгоритма ...

$$
G\left(\Omega_{u}, \Omega_{v}\right)=G_{00}-0.5\left(\alpha^{2} \Omega_{u}^{2}+\beta^{2} \Omega_{v}^{2}\right) .
$$

Здесь $\varphi$ - угол поворота, приводящий к канонической форме

$$
\operatorname{tg}(2 \varphi)=\frac{G_{12}}{G_{11}-G_{22}}
$$

$\alpha^{2}=\lambda_{1}, \beta^{2}=\lambda_{2}$, где $\lambda_{1}, \lambda_{2}-$ корни уравнения

$$
\left|\begin{array}{cc}
G_{11}-\lambda & G_{12} \\
G_{12} & G_{22}-\lambda
\end{array}\right|=0 .
$$

Причем $\lambda_{1}+\lambda_{2}=G_{11}+G_{22}, \lambda_{1} \lambda_{2}=G_{11} G_{22}-G_{12}^{2}$. Из пары соотношений для $S\left(\theta_{x}, \theta_{y}\right) \leftrightarrow G\left(\omega_{x}, \omega_{y}\right)$ (3) следует, что

$$
\begin{gathered}
G_{11}=\iint \theta_{x}{ }^{2} S\left(\theta_{x}, \theta_{y}\right) d \theta_{x} d \theta_{y}=\int \theta_{x}{ }^{2} S\left(\theta_{x}\right) d \theta_{x}, \\
G_{22}=\iint \theta_{y}{ }^{2} S\left(\theta_{x}, \theta_{y}\right) d \theta_{x} d \theta_{y}=\int \theta_{y}{ }^{2} S\left(\theta_{y}\right) d \theta_{y},(8) \\
G_{12}=\iint \theta_{x} \theta_{y} \cdot S\left(\theta_{x}, \theta_{y}\right) d \theta_{x} d \theta_{y} .
\end{gathered}
$$

Таким образом, можно перейти от повернутого изображения объекта к виду, аналогичному на рис. 16, используя (6), (7), (8), и привести АКФ к виду $S\left(\theta_{x}, \theta_{y}\right) \approx S\left(\theta_{x}\right) S\left(\theta_{y}\right)$.

Если в качестве эталонной оценки взять оценку максимального правдоподобия [16, 17], то используя численный алгоритм Ньютона - Рафсона, можно записать выражение для оценки в виде:

$$
\begin{aligned}
& \theta_{m x}=\theta_{f x}-\left.\left(\frac{d_{x}(\vec{\theta}) \quad d_{y y}(\vec{\theta})-d_{y}(\vec{\theta}) \quad d_{x y}(\vec{\theta})}{d_{x x}(\vec{\theta}) d_{y y}(\vec{\theta})-\left(d_{x y}(\vec{\theta})\right)^{2}}\right)\right|_{\theta=\theta_{f}} \\
& \theta_{m y}=\theta_{f y}-\left.\left(\frac{d_{y}(\vec{\theta}) d_{x x}(\vec{\theta})-d_{x}(\vec{\theta}) \quad d_{x y}(\vec{\theta})}{d_{x x}(\vec{\theta}) d_{y y}(\vec{\theta})-\left(d_{x y}(\vec{\theta})\right)^{2}}\right)\right|_{\theta=\theta_{f}}
\end{aligned}
$$

где

$$
\begin{aligned}
& d_{x}(\vec{\theta})=\partial M(\vec{\theta}) / \partial \theta_{x}, \\
& d_{y}(\vec{\theta})=\partial M(\vec{\theta}) / \partial \theta_{y}, \\
& d_{x x}(\vec{\theta})=\partial^{2} M(\vec{\theta}) / \partial \theta_{x}^{2}, \\
& d_{y y}(\vec{\theta})=\partial^{2} M(\vec{\theta}) / \partial \theta_{y}^{2}, \\
& d_{x y}(\vec{\theta})=\partial^{2} M(\vec{\theta}) /\left(\partial \theta_{x} \partial \theta_{y}\right) .
\end{aligned}
$$

Для

$$
\text { факторизуемых }
$$

АКФ $S\left(\theta_{x}, \theta_{y}\right) \approx S\left(\theta_{x}\right) S\left(\theta_{y}\right)$ можно считать, что и ЛФОП $M(\vec{\theta})=M\left(\theta_{x}\right) M\left(\theta_{y}\right)$. Тогда смешанные производные обращаются в нуль вблизи точки максимума и получаются две раздельные оценки. Если ЛФОП не факторизуется, то раздельные оценки будут квазиоптимальными. В дальнейшем будем считать, что производится раздельная оценка положения фрагмента $\theta_{m x}, \theta_{m y}$.

\section{1. Алгоритмы дискриминаторов}

Рассмотрим типичные дискриминационные алгоритмы, приближенно вычисляющие раздельные ОМП

I. Конечно-разностный дискриминатор

$$
\begin{aligned}
& \lambda_{m}\left(\theta_{f}, \delta\right)=\frac{M\left(\theta_{f}+\delta\right)-M\left(\theta_{f}-\delta\right)}{M\left(\theta_{f}+\delta\right)-2 M\left(\theta_{f}\right)+M\left(\theta_{f}-\delta\right)}= \\
& =\frac{q\left[\Psi^{-}\left(\theta_{f}, \delta\right)\right]+\left[N^{-}\left(\theta_{f}, \delta\right)\right]}{q\left[\Psi^{+}\left(\theta_{f}, \delta\right)-2 S\left(\theta_{f}\right)\right]+\left[N^{+}\left(\theta_{f}, \delta\right)-2 N\left(\theta_{f}\right)\right]} .(9)
\end{aligned}
$$

II. Суммарно-разностный дискриминатор

$$
\begin{gathered}
\lambda_{m}\left(\theta_{f}, \delta\right)=\frac{M\left(\theta_{f}+\delta\right)-M\left(\theta_{f}-\delta\right)}{M\left(\theta_{f}+\delta\right)+M\left(\theta_{f}-\delta\right)}= \\
=\frac{q\left[\Psi^{-}\left(\theta_{f}, \delta\right)\right]+\left[N^{-}\left(\theta_{f}, \delta\right)\right]}{q\left[\Psi^{+}\left(\theta_{f}, \delta\right)\right]+\left[N^{+}\left(\theta_{f}, \delta\right)\right]} .
\end{gathered}
$$

III. Дискриминатор с АРУ

$$
\begin{gathered}
\lambda_{m}\left(\theta_{f}, \delta\right)=\frac{M\left(\theta_{f}+\delta\right)-M\left(\theta_{f}-\delta\right)}{M\left(\theta_{f}\right)}= \\
=\frac{q\left[\Psi^{-}\left(\theta_{f}, \delta\right)\right]+\left[N^{-}\left(\theta_{f}, \delta\right)\right]}{q\left[S\left(\theta_{f}\right)\right]+\left[N\left(\theta_{f}\right)\right]},
\end{gathered}
$$

где

$$
\begin{aligned}
& \Psi^{-}\left(\theta_{f}, \delta\right)=S\left(\theta_{f}+\delta\right)-S\left(\theta_{f}-\delta\right), \\
& \Psi^{+}\left(\theta_{f}, \delta\right)=S\left(\theta_{f}+\delta\right)+S\left(\theta_{f}-\delta\right), \\
& N^{-}\left(\theta_{f}, \delta\right)=N\left(\theta_{f}+\delta\right)-N\left(\theta_{f}-\delta\right), \\
& N^{+}\left(\theta_{f}, \delta\right)=N\left(\theta_{f}+\delta\right)+N\left(\theta_{f}-\delta\right) .
\end{aligned}
$$

Алгоритмы (9)-(11) не требуют дифференцируемости ЛФОП.

Предположим, что в алгоритмах (9)-(11) ОСШ $q \gg 1$. Тогда шумовой компонентой можно пренебречь, и можно провести анализ двумерной оценки $\theta_{m x}, \theta_{m y}$. 


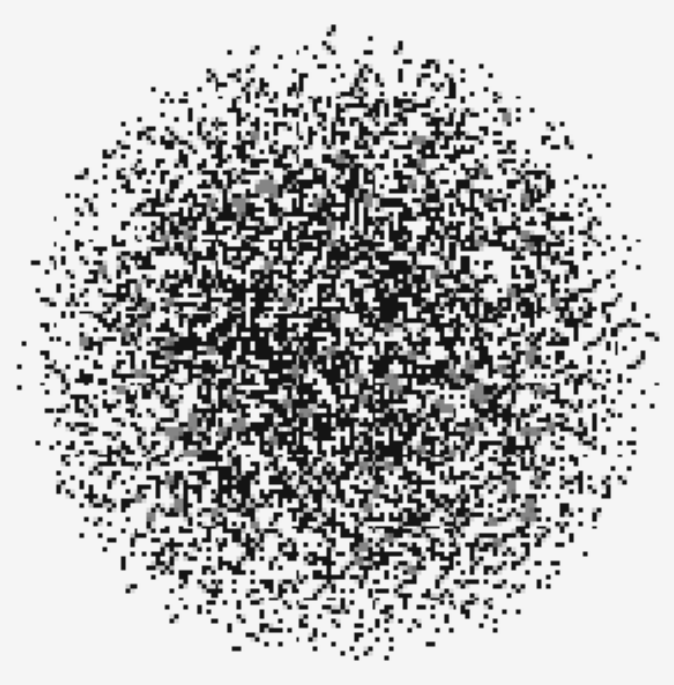

Puc. $3 a$

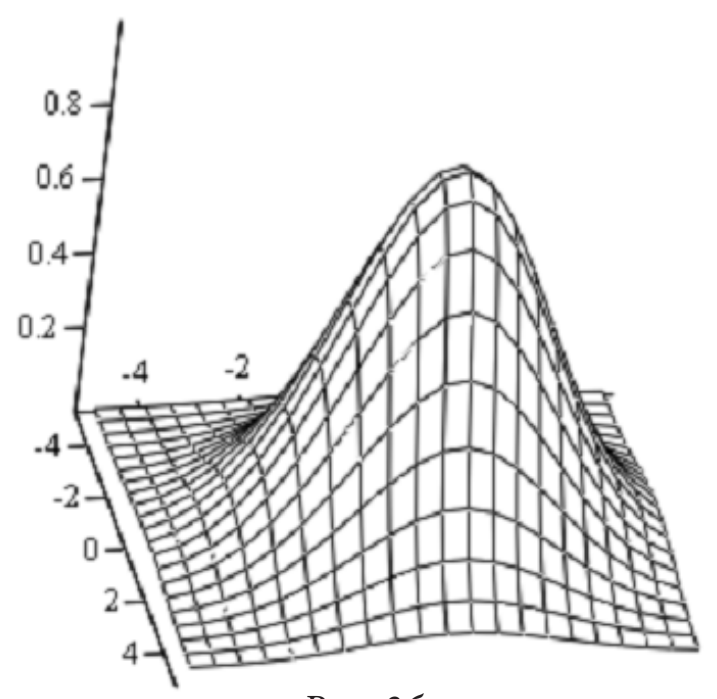

Puc. 36

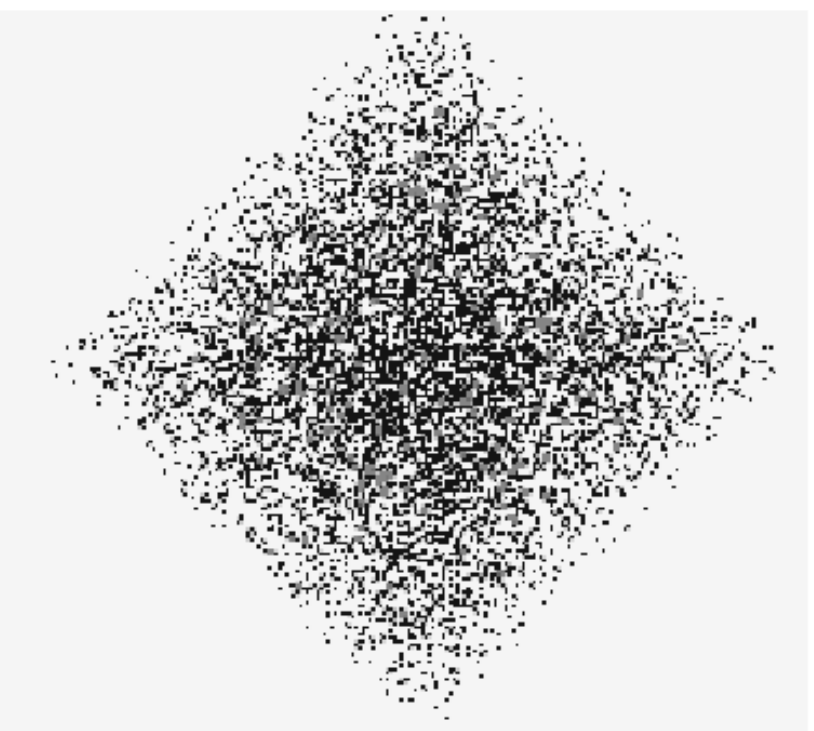

Puc. $4 a$

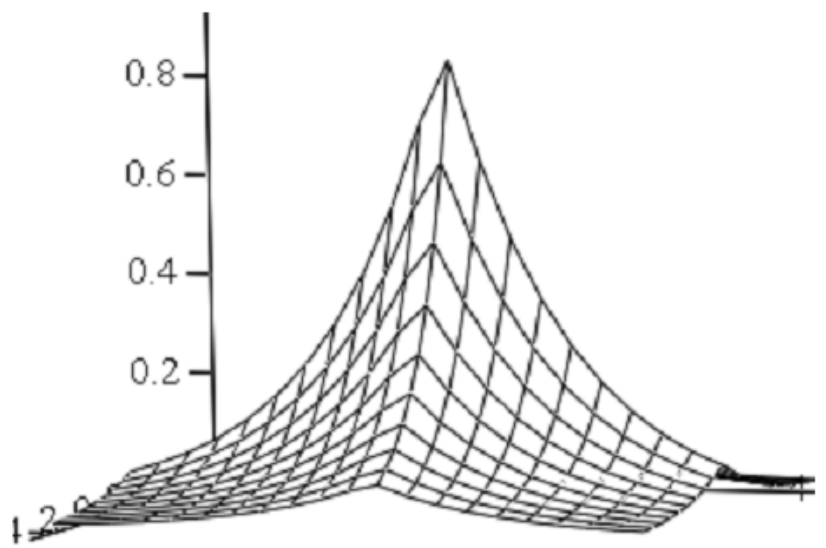

Puc. 46

На рис. За и рис. 4а представлены модельные фрагменты изображений, а на рис. 36 и на рис. 46 - их АКФ.

Фрагмент За и АКФ 36 соответствуют дифференцируемой модели поля, 4а и 46 -недифференцируемой.

Применение алгоритма (10) по дискретному изображению позволило при отсутствии шумов получить отличие оценки от истинного значения порядка $10^{-2}-10^{-3}$. В то время как поиск максимума по дискретной сетке дает точность оценки в 1 пиксель. Установлено, что для недифференцируемой модели АКФ (рис. 4б) межпиксельная интерполяция не повышает точности оценки.

\section{2. Анализ распределения}

Как следует из формул (9)-(11) дискриминационная статистика представляется в виде

$$
\lambda_{m}=\frac{\xi_{1}}{\xi_{2}}=\frac{q M_{1}+U_{1}}{q M_{2}+U_{2}} .
$$

Здесь $U_{1} \sim N\left(0, D_{1}\right), U_{2} \sim N\left(0, D_{2}\right)$, а $M_{2}-$ детерминированные компоненты числителя и знаменателя формул (9)-(11), зависящие от значений АКФ. $D_{1}, D_{2}$ - дисперсии случайных компонент $U_{1}, U_{2}$. Для алгоритмов (9)(11) гауссовские случайные величины $U_{1}, U_{2}$ независимы между собой.

Вводя обозначения $t=\frac{\xi_{2}}{\sqrt{D_{2}}}, \quad \mu=\frac{D_{2}}{D_{1}}$, $\chi=\frac{M_{2}}{\sqrt{D_{2}}}, \quad \frac{M_{1}}{\sqrt{D_{2}}}=\frac{M_{1}}{M_{2}} \frac{M_{2}}{\sqrt{D_{2}}}=\chi \lambda_{0}, \quad \frac{M_{1}}{M_{2}}=\lambda_{0}$, 
Характеристики двумерной оценки положения фрагмента изображения на основе алгоритма ...

можно записать плотность вероятности $\lambda_{m}$ (12) как

$$
\begin{gathered}
W(\lambda, q)=\frac{\sqrt{\mu}}{2 \pi} \times \\
\times \int_{-\infty}^{\infty}|t| \exp \left\{-\frac{\left[(t-q \chi)^{2}+\mu\left(\lambda t-q \chi \lambda_{0}\right)^{2}\right]}{2}\right\} d t .
\end{gathered}
$$

Для анализа влияния рассогласования по параметрам сигнала и приёмника и величины ОСШ на характеристики распределение $\lambda_{m}$ были построены соответствующие зависимости. На рис. 5 представлена зависимость распределения от параметра рассогласования различных ОСШ $q=2,3,5$, и параметре рассогласования $\theta_{f}=1$. Здесь же приведена точками экспериментальная оценка плотности вероятности $Y(\lambda)$. Экспериментальная плотность вероятности $Y(\lambda)$ получена ядерным сглаживанием с гауссовской весовой функцией выборки статистики $\lambda_{m}$ (оценка Парзена), объемом $n=3000$ [16,17]. Соответствие распределений показано не только графически, но и по критерию Колмогорова при $\alpha \leq 0.1$.

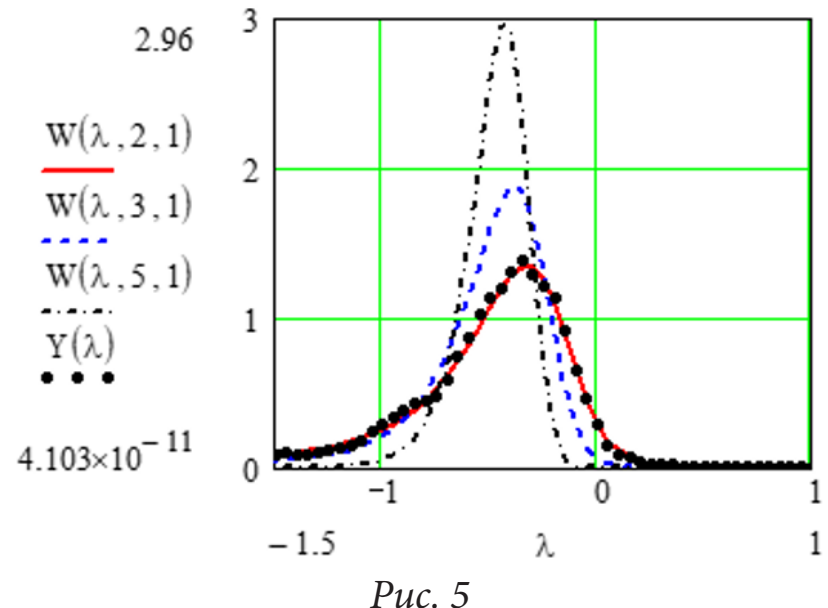

Для распределения (13) можно получить явный аналитический вид в двух случаях: ОСШ $q=0$ и $q \gg 1$. В первом случае $W(\lambda, q=0)=\frac{\sqrt{\mu}}{\pi} \frac{1}{1+\mu \lambda^{2}}$. Это распределение Коши.

Во втором случае после асимптотического интегрирования по методу Лапласа [18] получаем

$$
\begin{gathered}
W(\lambda, q)=\frac{\sqrt{\mu} q \chi}{\sqrt{2 \pi}} \cdot \frac{\left|1+\mu \lambda \lambda_{0}\right|}{\left(1+\mu \lambda^{2}\right)^{3 / 2}} \times \\
\quad \times \exp \left(-\frac{1}{2} q^{2} \chi^{2} \frac{\mu\left(\lambda-\lambda_{0}\right)^{2}}{1+\mu \lambda^{2}}\right) .
\end{gathered}
$$

Из анализа формул (13), (14) и статистического моделирования следует, что при конечных ОСШ оценка является несостоятельной из-за наличия «тяжёлых хвостов» распределения, которые приводят к бесконечной дисперсии.

\section{ЗАКЛЮЧЕНИЕ}

Исследована возможность оценки местоположения фрагмента изображения в кадре с помощью дискриминаторов. Для изображений объектов с факторизуемыми автокорреляционными функциями рассмотрены типовые одномерные дискриминационные алгоритмы. Показано, что распределение процесса на выходе некоторых типов дискриминаторов является существенно негауссовским, оно имеет «тяжелые хвосты». Исследовано поведение этого распределения в зависимости от отношения сигнал/шум, величины рассогласования по оцениваемому параметру принятого и опорного сигналов. Применение дискриминаторов к дискретному изображению позволяет уменьшить число операций в десятки раз по сравнению с известными сеточными методами оптимизации.

\section{СПИСОК ЛИТЕРАТУРЫ}

1. Дворкович, В. П. Цифровые видеоинформационные системы (теория и практика) / В. П. Дворкович, А. В. Дворкович - Москва: Техносфера, 2012. - 1008 с.

2. Дворкович, В. П. Измерения в видеоинформационных системах (теория и практика) / В. П. Дворкович, А. В. Дворкович - Москва: Техносфера, 2015. - 783 с.

3. Системы автоматического обнаружения и сопровождения объектов. Обработка изображений и управление / Б. А. Алпатов [и др.]. - Москва: Радиотехника, 2008. - 176 с. 
4. Васильев, К. К. Статистический анализ последовательностей изображений/ К. К. Васильев, В. Р. Крашенинников. - Москва: Радиотехника, 2017. - 248 с.

5. Radchenko, Yu. S. Methods for Detecting of Structural Changes in Computer Vision Systems / Yu. S. Radchenko, A. V. Bulygin// Computer Vision in Control Systems-1: International Publishing - Switzerland: 2015. - Chapter 3. P. 59-90.

6. de Queiroz, R. L. Motion-Compensated Compression of Dynamic Voxelized Point Clouds / R. L. de Queiroz, P. A. Chou //IEEE Transactions on Image Processing, 2017. - V. 26, № 8 . - P. 3886-3895.

7. Ouzir, N. Motion Estimation in Echocardiography Using Sparse Representation and Dictionary Learning / N. Ouzir [and etc] //IEEE Transactions on Image Processing, 2018. - V. 27, №1. - P. 64-77.

8. Cuрота, A. А. Адаптивные алгоритмы построения сверхразрешения на основе обработки последовательности изображений / А. А. Сирота, А. Ю. Иванников // Оптический журнал. - 2017. - Т. 84, № 5. - С. 38-45.

9. Chao, R. Single Image Super-Resolution via Adaptive High-Dimensional Non-Local Total Variation and Adaptive Geometric Feature / R. Chao, He Xiaohai, N. Truong // IEEE Transactions on Image Processing, 2017. -V. 26, № 1. P. 90-106.

10. Антонова, Г. М. Сеточные методы равномерного зондирования для исследования и оптимизации динамических стохастических систем / Г. М. Антонова. - Москва : Физматлит, 2007. - 224 с.

Радченко Ю. С. - д-р физ.-мат. наук, профессор кафедры радиофизики, физический факультет, Воронежский государственный университет.

E-mail: ysradchenko@yandex.ru

Машарова О. А. - аспирант кафедры радиофизики, физический факультет, Воронежский государственный университет.

E-mail: masharova.ol@yandex.ru
11. Оценивание дальности и скорости в радиолокационных системах. / под ред. А. И. Канащенкова и В. И. Меркулова. - Москва: Радиотехника, 2004. - Ч. 1. - 312 с.

12. Радиоавтоматика: учебное пособие / В. А. Бессекерский, [и др.]; под ред. В. А. Бессекерского. - Москва: Высш. Школа, 1985. $271 \mathrm{c}$.

13. Машарова, О. А. О законе распределения сигналов рассогласования некоторых типов дискриминаторов / О. А. Машарова, Ю. С. Радченко// Физические основы приборостроения. - 2017. - Т. 6, № 2 (24). - С. 90-97.

14. Прохоров, Ю. В. Теория вероятностей (Основные понятия. Предельные теоремы. Случайные процессы) / Ю. В. Прохоров, Ю. А. Розанов. - Москва: Физматлит, 1973. $494 \mathrm{c}$.

15. Тыртыциников, Е. Е. Матричный анализ и линейная алгебра / Е. Е. Тыртышников. - Москва: Физматлит, 2007. - 480 с.

16. Сuрота, A. A. Методы и алгоритмы анализа данных и их моделирование в MATLAB / А. А. Сирота. - СПб. : БХВ-Петербург, 2016. - 384 с.

17. Лагутин, М. Б. Наглядная математическая статистика / М. Б. Лагутин. - Москва: БИНОМ. Лаборатория знаний, 2007. - 472 с

18. Олвер, Ф. Введение в асимптотические методы и специальные функции / Ф. Олвер; [пер. с англ. Ю. А. Брычкова, под ред. А. П. Прудникова]. - Москва: Физматлит, 1990. - 528 c.

Radchenko Y. S. - Dr. Sci. (Phys. -Math.), professor of radio physics department of the Voronezh State University.

E-mail: ysradchenko@yandex.ru

Masharova O. A. - graduate student of radio physics department of the Voronezh State University.

E-mail: masharova.ol@yandex.ru 